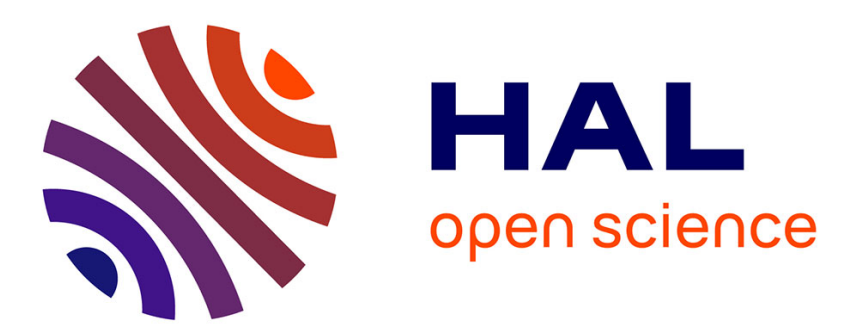

\title{
Tuning the gains of haptic couplings to improve force feedback stability in nanorobotics
}

\author{
Aude Bolopion, Barthélemy Cagneau, Sinan Haliyo, Stéphane Régnier
}

\section{To cite this version:}

Aude Bolopion, Barthélemy Cagneau, Sinan Haliyo, Stéphane Régnier. Tuning the gains of haptic couplings to improve force feedback stability in nanorobotics. 2009 IEEE International Conference on Robotics and Automation (ICRA), May 2009, Kobe, Japan. pp.4320-4325, 10.1109/ROBOT.2009.5152270 . hal-02912220

\section{HAL Id: hal-02912220 \\ https://hal.science/hal-02912220}

Submitted on 5 Aug 2020

HAL is a multi-disciplinary open access archive for the deposit and dissemination of scientific research documents, whether they are published or not. The documents may come from teaching and research institutions in France or abroad, or from public or private research centers.
L'archive ouverte pluridisciplinaire HAL, est destinée au dépôt et à la diffusion de documents scientifiques de niveau recherche, publiés ou non, émanant des établissements d'enseignement et de recherche français ou étrangers, des laboratoires publics ou privés. 


\title{
Tuning the gains of haptic couplings to improve force feedback stability in nanorobotics
}

\author{
Aude Bolopion, Barthélemy Cagneau, D. Sinan Haliyo, and Stéphane Régnier
}

\begin{abstract}
This paper deals with the problem of bilateral haptic control in nanorobotics. At this scale, a human operator cannot interact directly with objects. He needs special tools manipulated through robotic systems. Therefore, force feedback devices are the only solution to provide him a sense of touch. However, the quality of the rendering strongly influences his ability to perform a given task. Stability is the main requirement that the system must fulfil to be usable. As the choice of the controller and its tuning are critical issues, a general method to tune the parameters of two haptic controllers is presented. A theoretical study is carried out and the methodology is validated with an experiment composed of several phases with high dynamic phenomena. Intrinsic limitations of the two controllers are also pointed out.
\end{abstract}

\section{INTRODUCTION}

The importance of microscale and nanoscale robotic systems is growing and new application fields are emerging. From mini-invasive surgery to ultra-high density storage devices and sensor networks for environmental monitoring, micro and nano technologies are becoming part of our every day life [1]. However, at this scale, manipulation of objects is still a challenge.

At the submicrometer scale, it is necessary to overcome a critical problem before performing any task: human operators cannot interact directly with the environment. Consequently, special tools are needed to allow operations and to provide visual and tactile feedback. However, the resolution of optical microscopes is limited. Scanning Probe Microscopes (SPM) provide greater accuracy, but it is not possible to use them to capture images and manipulate objects simultaneously. Scanning Electron Microscopes (SEM) do not provide realtime images. That is why haptic coupling appears to be an interesting solution to establish a link between these two worlds, ([2], [3]). Indeed, even elementary tasks lithography or indentation, or characterization of objects' mechanical properties cannot be performed without visual or haptic feedback [4], [5], [6].

Based on dimensional analysis, [7] presents a method to select the coefficients that are used to scale forces and positions for haptic coupling. However, practical issues, like limited workspace and range of forces that can be felt by a human operator while manipulating a haptic device (typically from 0.1 to $10 \mathrm{~N}$ ), will prevent using this kind of reasoning.

Other works on coupling issues include [8], which also proposes a method to derive scaling factors based on Llewelyn's criteria. A passivity controller is implemented to assure

\footnotetext{
Authors are with Institut des Systèmes Intelligents et de Robotique, Université Pierre et Marie Curie, CNRS UMR 7222, 4 Place Jussieu, 75252 Paris Cedex, France \{bolopion, cagneau, haliyo, regnier\}eisir.fr
}

stability. However, it has been tested only on virtual environments and no method to tune the gains of the controller is provided. [9] also presents very interesting results with the cancellation of the cantilever's stiffness on the rendered forces and more detailed conclusions about the passivity controller.

In this paper, we will present two different control schemes. To tune the gains of the controllers, an original method that can be adapted to most of the usual systems is used. It considers simple inequalities between the parameters of the controllers so that they can be chosen according to simple relations. Stability is the main requirement that will be considered in deriving these relations. Compared to [10], the resolution of the force sensor has been increased, allowing users to feel forces in the range of nanonewton (instead of micronewton). Morevoer, we do present rules to tune the gains, and experimental results for cantilevers of different stiffnesses.

This paper is organized as follows: in section II, we present the experiments and the experimental setup that we will use to validate the control schemes; then, the most intuitive control scheme, the Direct Force Feedback is presented in section III. We will show its limitations in our context. Section IV introduces another control scheme, the ForcePosition control scheme, and an original method for choosing the controllers' gains. Throughout this paper, experimental results will be presented to underscore our conclusions.

\section{EXPERIMENTAL SETUP}

\section{A. Coupling validation's protocol}

To compare the performances of the different couplings and the influence of the controllers' gains, we will perform a basic one-dimensional experiment. It consists of approaching the substrate with the probe, applying a force and then retracting. The well-known plot representing the force applied on the cantilever as a function of the distance from the substrate is given in figure 1(a). The corresponding plot of force applied on the cantilever as a function of time is given in figure 1(b). This is what the operator should ideally feel. Two discontinuous phenomena (referenced as $\mathrm{A}$ and $\mathrm{E}$ on the plots) are respectively representative of the instant when the cantilever is attracted by the substrate (pull-in) and when the contact is broken (pull-off). Two kinds of forces are present: the very first ones (B) and the last ones (D) are attractive (just after the pull-in and just before the pull-off), while the ones in between are repulsive $(\mathrm{C})$. Since attractive forces and discontinuous phenomena are present at nanoscales, the coupling will have to be designed and tuned taking these 
characteristics into consideration. Indeed, it has to remain stable and the user must be able to feel these phenomena.

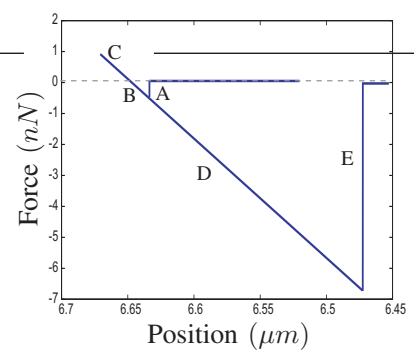

(a)

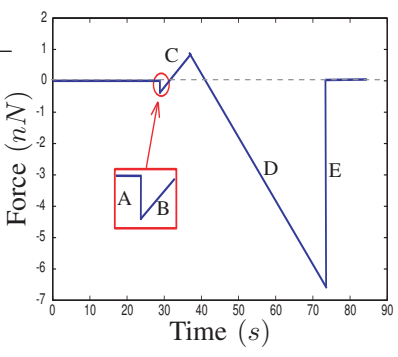

(b)
Fig. 1. Approach-retract curve

As the control scheme used depends on the master and slave devices, the experimental setup has to be detailed before performing any analysis.

\section{B. Experimental platform}

The experimental platform used to validate the coupling is depicted on figure 3 .

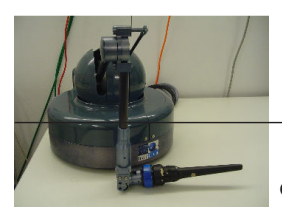

Fig. 2. Haptic interface

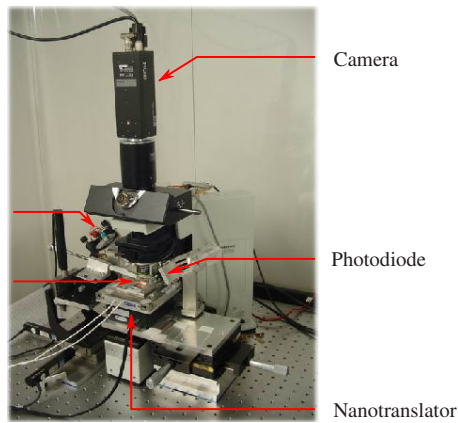

Fig. 3. Experimental setup
It is composed of a nanotranslator which can move along the vertical direction.

The force applied on the cantilever is measured with a laser. The deflection is measured using a laser beam focused on the cantilever. The beam is reflected onto a photodiode. The normal force is computed as:

$$
F=k S V
$$

where $k$ and $S$, the stiffness of the cantilever and the sensitivity of the photodiode, are calibrated [11]. $V$ is the output voltage of the photodiode.

As highlighted in equation (1), the force depends on $k$. Therefore, the coupling will be influenced by this factor. However, the proposed method must be adapted for cantilevers of any stiffness.

The available haptic device is a Virtuose manufactured by Haption $^{1}$. This arm, represented on figure 2, allows 3 degrees of freedom of force feedback.

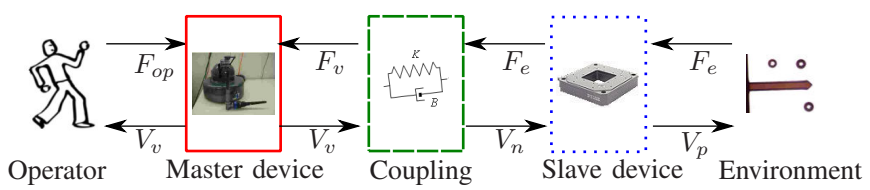

Fig. 4. Power flow between the subsystems

\section{Power flow}

Figure 4 summarizes the power flow between the different subsystems. The inputs are:

- $F_{e}$ : force applied by the substrate on the cantilever

- $F_{o p}$ : force applied by the operator on the master arm

The set of variables that corresponds to the outputs is:

- $V_{p}$ : velocity of the cantilever in the operational space

- $V_{v}$ : velocity of the master arm in the operational space

$F_{v}$ is the force fed back to the user, through the haptic interface, and $V_{n}$ is the desired nanotranslator velocity. The subsystems' inputs and outputs are the only assumptions we make in designing the control scheme. However, they are not really restrictive since many teleoperation systems presented in literature fulfill these requirements.

\section{DIRECT FORCE FEEDBACK}

Direct Force Feedback ( $D F F$ ) control scheme seems to be the most intuitive formulation for providing amplified forces to the operator. It is depicted on figure 5. The blocks and

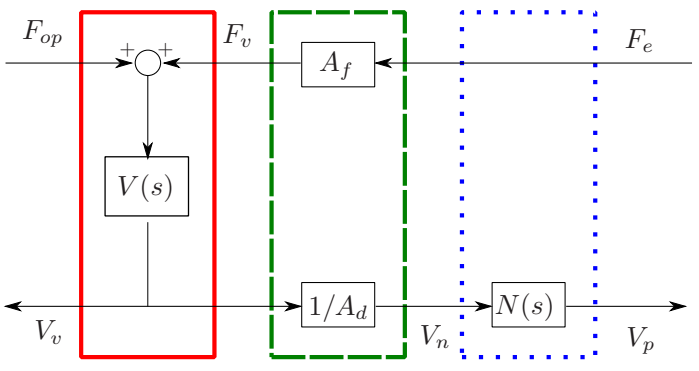

Fig. 5. Control scheme for $D F F$.

power flows defined on figure 4 are clearly identified.

Master (Virtuose) and slave (nanotranslator) continuoustime transfer functions are modeled as follows:

$$
\begin{aligned}
& V(s)=\left[\left(B_{v}+M_{v} s\right)\right]^{-1} \\
& N(s)=\left[\left(1+\tau_{1} s\right)\left(1+\tau_{2} s\right)\right]^{-1}
\end{aligned}
$$

where $s$ is the Laplace variable. Numerical values of the characteristic parameters have been identified as:

$$
\begin{array}{lll}
M_{v}=0.4 k g & ; & B_{v}=0.1 N . s . m^{-1} \\
\tau_{1}=1.35 \times 10^{-3} s & ; & \tau_{2}=0.57 \times 10^{-3} s
\end{array}
$$

where $M_{v}$ and $B_{v}$ are respectively the inertia and damping of the Virtuose. $\tau_{1}$ and $\tau_{2}$ are time constants of the nanotranslator. $A_{f}$ and $A_{d}$ are respectively the force and velocity scaling factors. These coefficients are necessary to adjust the scale difference between macro- and nano-worlds.

\footnotetext{
${ }^{1}$ http://www.haption.com/
} 
The main advantage of this control scheme is that it requires only these two parameters to achieve $D F F$ control. On the other hand, the system's stability will entirely depend on these coefficients.

\section{A. Routh stability criterion}

The slave device interacts with a remote environment, which will influence the stability of the coupling. Therefore, it has to be considered while performing stability analysis. Two approaches can be used. The first is to consider a method that does not require modeling the environment (e.g., methods based on passivity study). However, it is more conservative, and not useful in pointing out limitations as a function of the cantilever' and the environment's characteristics. The second approach is to model the environment. This is what we will do to derive limitations of the control scheme for our particular application.

The environment will be modeled with respect to assumption (1).

Assumption 1: The slave device is linked with its environment through a spring of stiffness $k_{c}$ (the cantilever). The contact between the cantilever and the substrate is modeled as a spring $k_{s}$. These two serial springs are linked such that the equivalent spring $k_{e q}$ is:

$$
\frac{1}{k_{e q}}=\frac{1}{k_{s}}+\frac{1}{k_{c}}
$$

Considering this assumption and the control scheme depicted on figure 5 , it is possible to determine the transfer function between $F_{o p}$ and $V_{v}$. Since the system is linear time invariant (LTI), the Routh-Hurwitz criterion can be applied to the transfer function. A necessary and sufficient condition of stability is derived. It leads to the following inequality:

$$
R=\frac{A_{f}}{A_{d}} \leq \frac{\gamma}{k_{e q}}=R_{\max }
$$

where $\gamma=\frac{B_{v}\left(\tau_{1}+\tau_{2}\right)\left[M_{v}^{2}+M_{v} B_{v}\left(\tau_{1}+\tau_{2}\right)+B_{v}^{2} \tau_{1} \tau_{2}\right]}{\left[M_{v}\left(\tau_{1}+\tau_{2}\right)+B_{v} \tau_{1} \tau_{2}\right]^{2}}$

As $\gamma$ only depends on the system's parameters, for a given environment, equation (5) shows that the system's stability only depends on the ratio $R=\frac{A_{f}}{A_{d}}$.

The system's stability is assured by selecting $R \leq R_{\max }$ with respect to equation (5). $R_{\max }$ will also determine the closed loop performances in terms of force/velocity scaling. However, it is obvious that the ratio $R_{\max }$, inversely proportional to $k_{e q}$, is decreasing sharply when environmental stiffness increases. The next subsection will provide a compromise between stability and the values of the scaling factors through experimental examples.

For stability issues, the worst case is when equivalent stiffness is the highest. Using Hertz's theory [12], it can be shown that it corresponds to $k_{e q}=k_{c}$. Therefore, in the following sections we will use this approximation.

\section{B. Experimental results}

We used a cantilever with $k_{c}=2.4 N \cdot m^{-1}$ so that:

$$
R_{\max }=21.7
$$

The experiment described in section II-A is performed with the following values:

$$
\left.\begin{array}{l}
A_{d}=0.05 \cdot 10^{6} \\
A_{f}=0.12 \cdot 10^{6}
\end{array}\right\} \Rightarrow R=2.4<R_{\max }
$$

$A_{d}$ is chosen according to the master and slave motion ranges. The value $A_{d}=0.05 \cdot 10^{6}$ is such that the master motion range, which is $25 \mathrm{~cm}$, corresponds to a displacement of $5 \mu \mathrm{m}$ for the slave. This gives a good compromise between ease of manipulation and precision. This value will be kept constant for all experiments, to allow a better comparison between them. In this first experiment, $A_{f}$ is chosen so that the Routh criterion given in equation (5) is satisfied. Stability will be studied with respect to this factor in next paragraphs. The results we have obtained on figure 6 confirm

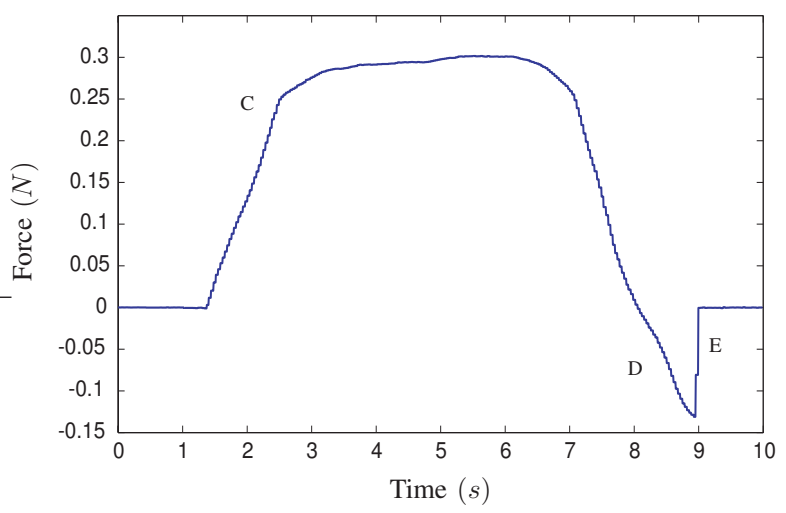

Fig. 6. Approach-retract curve for a cantilever of stiffness $k_{c}=$ $2.4 N . m^{-1}$, using $A_{d}=0.05 \cdot 10^{6}, A_{f}=0.12 \cdot 10^{6}$. R=2.4.

our predictions. Indeed, this figure represents the magnitude of the forces sent back to the user. During the first stage (C), the operator applies forces on the substrate. The maximum value of the forces applied is $0.30 \mathrm{~N}$. While retracting, he must counterbalance the forces resulting from adhesion effects (D). A force equal to $-0.13 N$ is necessary to release the cantilever from the substrate $(\mathrm{E})$.

Even if the system remains stable as expected, force feedback does not allow an operator to distinguish the different stages described on section II-A (attraction, repulsion, pulloff, etc.). Therefore, it might be interesting to increase $A_{f}$ in order to provide better force feedback to the user. The pull-in phenomenon is not visible on the plot. Indeed, to avoid time consuming operations, we chose $A_{d}=0.05 \cdot 10^{6}$. With this value, the velocity of the nanotranslator is too high compared to the dynamics of the pull-in effect, and so it could not be reflected to the user.

During the second experiment we performed, the value of $A_{d}$ remained the same, but $A_{f}$ was increased, so that $A_{f}=6.0 \cdot 10^{6}$. With these values:

$$
R=120>R_{\max }
$$

Equation (8) shows that the Routh criterion is no longer satisfied. The system is thus predicted to be unstable. The results we have obtained are plotted on figure 7. Remarkably, the forces have been amplified compared to the forces of the 


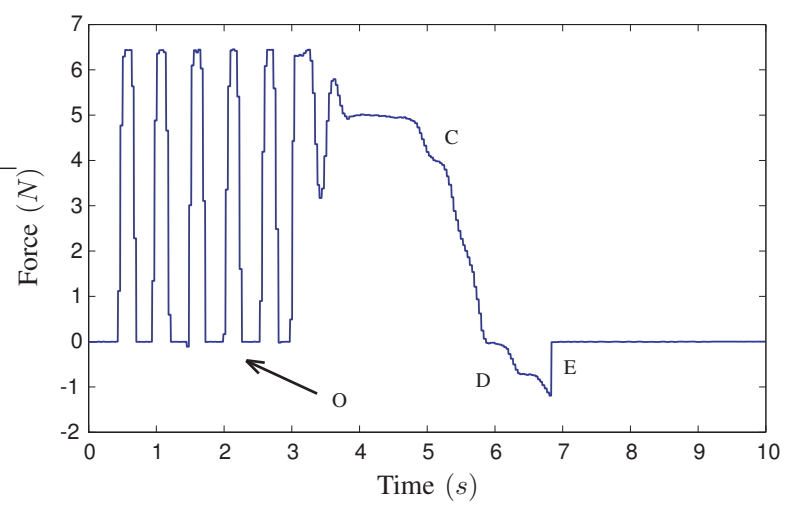

Fig. 7. Approach-retract curve for a cantilever of stiffness $k_{c}=$ $2.4 N \cdot m^{-1}$, using $A_{d}=0.05 \cdot 10^{6}, A_{f}=6 \cdot 10^{6} . \mathrm{R}=120$.

figure 6. It is then easier for the user to detect repulsive and attractive forces $(\mathrm{C}, \mathrm{D})$ and the high variation of the forces due to the pull-off $(\mathrm{E})$. This result shows that force reflection has been improved by modifying the value of $A_{f}$.

However, the stability of the system is affected. When the cantilever establishes the contact with the substrate, it creates high amplitude oscillations in the system $(\mathrm{O})$. These oscillations are very disturbing for the user who must compensate for these perturbations. He has to act like a damper to absorb the amount of energy responsible for instability.

\section{FORCE-POSITION CONTROL}

All studies on this coupling will be conducted using a discrete time variable $z$. Indeed, contrary to the $D F F$ control scheme, FP involves numerical integrations which make the system very sensitive to the sampling period.

\section{A. Control scheme design}

It has been shown in section III that the DFF scheme presents limitations in terms of stability, depending on the desired scaling factors. To improve that issue, two controllers are added in the FP control scheme detailed on figure 8. $G_{n}$ and $C(z)$ are respectively proportional $(P)$ and proportionalintegral $(P I)$ controllers. Only $P$ and $P I$ controllers have been used. Indeed, their simple form highlights the influence of each gain on the system transparency and stability. A good understanding of the interactions between gain values and system performances is necessary to later design more complex control schemes. On-line gains tuning based on the rules that will be derived in next paragraphs will be studied in future works. The star superscript is for discrete parameters.

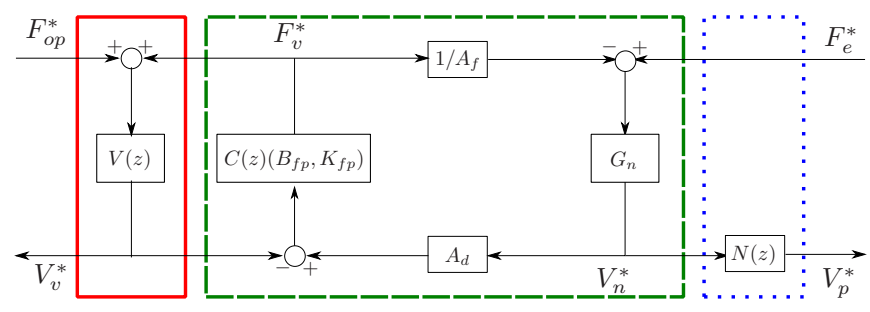

Fig. 8. Force-Position control scheme
$C(z)$ has been discretized using Tustin's approximation. This leads to:

$$
C(z)=B_{f p}+K_{f p} \frac{T_{e}(z+1)}{2(z-1)}
$$

In the following, $V(z)$ (resp. $N(z)$ ) will refer to the discrete time transfer function corresponding to $V(s)$ (resp. $N(s)$ ). They are such that:

$$
\begin{gathered}
V(z)=\frac{1}{B_{v}} \frac{1-\delta}{z-\delta} \text { with: } \delta=e^{-\frac{B_{v} T_{e}}{M_{v}}} \\
N(z)=1-\frac{\tau_{1}}{\tau_{1}-\tau_{2}} \frac{z-1}{z-e^{-\frac{T_{e}}{\tau_{1}}}}-\frac{\tau_{2}}{\tau_{2}-\tau_{1}} \frac{z-1}{z-e^{-\frac{T_{e}}{\tau_{2}}}}
\end{gathered}
$$

Compared to the DFF control scheme, the force fed back to the user, $F_{v}$, is computed with the $P I$ controller. The integral gain $K_{f p}$ and the proportional gain $B_{f p}$ can be used to modify the stiffness and damping of the rendered force. The gain $G_{n}$ is used to compute the desired velocity of the slave device.

\section{B. Determination of the control scheme parameters}

1) Scaling factors: As in the first control scheme, $A_{f}$ and $A_{d}$ depend on the user's requirements. Indeed, one might want a precise positioning, and/or important force feedback, according to the manipulation tasks. As in section III, we will choose $A_{d}=0.05 \cdot 10^{6}, A_{f}$ will be such that the forces sent back to the user are high enough for an untrained user to distinguish between the different phenomena encountered during the experiment.

2) Proportional controller $G_{n}$ : Problems of stability can be due to control schemes but also to numerical computation. Indeed, the force $F_{v}^{*}$ at time $k+1$ is determined using information of positions and velocities at time $k$ (see figure 8):

$$
\begin{aligned}
F_{v}^{*}(k+1) & =B_{f p} \Delta V(k)+K_{f p} \Delta X(k) \\
V_{n}^{*}(k+1) & =G_{n}\left[F_{e}^{*}(k)-\frac{1}{A_{f}} F_{v}^{*}(k)\right]
\end{aligned}
$$

where:

$$
\begin{aligned}
\Delta V(k) & =A_{d} V_{n}^{*}(k)-V_{v}^{*}(k) \\
\Delta X(k) & =A_{d} X_{n}^{*}(k)-X_{v}^{*}(k)
\end{aligned}
$$

Considering (12) and (13) and the fact that the position $X_{n}^{*}$ is computed using Tustin's discretization, when the tip is well above the substrate (no force applied on it, i.e., $F_{e}^{*}=0$ ), $F_{v}^{*}$ is given by:

$F_{v}^{*}(k+1)=\lambda_{1} F_{v}^{*}(k-1)+\lambda_{2} V_{v}^{*}(k)+\lambda_{3} K_{f p} A_{d}+\lambda_{4} K_{f p}$

where:

$$
\begin{aligned}
& \lambda_{1}=-G_{n}\left[\frac{B_{f p} A_{d}+K_{f p} A_{d} \frac{T_{e}}{2}}{A_{f}}\right] \\
& \lambda_{2}=-\left[B_{f p}+K_{f p} \frac{T_{e}}{2}\right] \\
& \lambda_{3}=\left[X_{n}^{*}(k-1)+\frac{V_{n}^{*}(k-1) T_{e}}{2}\right] \\
& \lambda_{4}=-\left[X_{v}^{*}(k-1)+\frac{V_{v}^{*}(k-1) T_{e}}{2}\right]
\end{aligned}
$$


Avoiding numerical instabilities leads to an upper boundary on $G_{n}$, a necessary condition for stability:

$$
\left|\lambda_{1}\right|<1 \Leftrightarrow G_{n}<\frac{A_{f}}{A_{d} K_{f p} \frac{T_{e}}{2}+A_{d} B_{f p}}
$$

However, for transparency reasons, $G_{n}$ has to be as high as possible. Otherwise, it leads to a tracking error on the slave side and thus to viscosity on the force fed back to the user.

3) Proportional integral controller $B_{f p}$ and $K_{f p}$ : In [13] a relation between $B_{f p}, K_{f p}$ and $T_{e}$ is derived to assure the stability of the system while in contact with an infinitely stiff environment. The same methodology applied to our system will be used here. As for the DFF control scheme, the system considered is LTI. The Tustin equation is used for the discretization.

The discrete time transfer function is:

$$
\frac{V_{v}^{*}}{F_{o p}^{*}}=\frac{V(z)}{1+V(z) C(z)}
$$

After applying the bilinear transformation $z=\frac{2+w T_{e}}{2-w T_{e}}$, the resulting characteristic equation becomes:

$$
b_{2} w^{2}+b_{1} w+b_{0}=0
$$

where:

$$
\begin{aligned}
b_{2} & =4 B_{v}(1+\delta)-4(1-\delta) B_{f p} \\
b_{1} & =(1-\delta)\left(4 B_{v}+4 B_{f p}-2 K_{f p} T_{e}\right) \\
b_{0} & =2 K_{f p} T_{e}(1-\delta)
\end{aligned}
$$

The Routh-Hurwitz criterion is achieved if and only if $b_{0}$, $b_{1}$ and $b_{2}$ have the same sign. Since $\delta<1$, (20) is always positive. Therefore, the system will be stable if and only if $b_{1}>0$ and $b_{2}>0$, which implies:

$$
\begin{aligned}
B_{v}+B_{f p} & >\frac{K_{f p} T_{e}}{2} \\
B_{v} \frac{1+\delta}{1-\delta} & >B_{f p}
\end{aligned}
$$

(21) is the same condition as that found in [13], and states that the stiffness of the coupling is bounded by the damping of the haptic interface and that added by the coupling. Moreover, if the sampling period increases, $K_{f p}$ must decrease for the same amount of damping to guarantee stability.

Using the first order Taylor development of $x \mapsto \exp (x)$ in the neighborhood of $0\left(\frac{B_{v} T_{e}}{M_{v}} \rightarrow 0\right.$ since $\left.T_{e} \rightarrow 0\right), \delta \approx$ $1-\frac{B_{v} T_{e}}{M_{v}}$. (22) can be approximated by:

$$
\frac{2 M_{v}}{T_{e}}>B_{v}+B_{f p}
$$

This highlights the fact that the maximum damping (and therefore, according to (21), the maximum stiffness) admissible is limited by the inertia of the master arm, and is inversely proportional to the sampling period $T_{e}$. This is a convincing argument of the importance of the sampling period for stable haptic feedbacks.

\section{Experimental results}

This subsection presents experimental results for the FP control scheme.

To validate the methodology described above, we will use the same parameters as for the $D F F$ control scheme. Taking the same cantilever as in section III-B $\left(k_{c}=2.4 N . \mathrm{m}^{-1}\right)$, we require the same scaling factors $\left(A_{d}=0.05 \cdot 10^{6}, A_{f}=\right.$ $\left.6 \cdot 10^{6}\right)$.

Based on conclusions of section IV-B, the following considerations have to be taken into account to choose the gains:

- $A_{f}$ and $A_{d}$ are determined by the user's requirements

- $K_{f p}$ has to be high to allow a stiff contact feeling. However, according to (21) and (23), it can not be higher than $\frac{4 M_{v}}{T_{e}^{2}}$

- $B_{f p}$ has to be low to allow for transparent feeling when no forces are applied on the cantilever. However, according to (21), it has to be higher than $\frac{K_{f p} T_{e}}{2}-B_{v}$.

The trade off between $K_{f p}$ and $B_{f p}$ greatly depends on the application. It is necessary to bear in mind the upper and lower boundaries but the values that match his requirements can be freely chosen.

- knowing all the other parameters, $G_{n}$ is given by (15) For our application, an admissible set of gains is: $B_{f p}=2.0$ N.s.m ${ }^{-1}, K_{f p}=100.0$ N.m ${ }^{-1}, G_{n}=$ $47.5 \mathrm{~m} \cdot \mathrm{N}^{-1} \cdot \mathrm{s}^{-1}$. It is necessary to verify experimentally that the method we described and the gains we chose are adapted to the stated problem.

The experimental results we have obtained are plotted on figure 9. They must be compared to those on figure 7 . It is obvious that the system remains stable during this experiment, unlike the $D F F$ control scheme. The small oscillations are induced by the virtual coupling. However, due to the limited bandwith of the haptic interface, this phenomenon is not disturbing for manipulations. As expected, the user is able to feel the pull-off phenomenon with a peak of amplitude $1 N$. Consequently, the method described through this paper allows the controller presented in section IV-A to be tuned efficiently. To prove the robustness of our approach

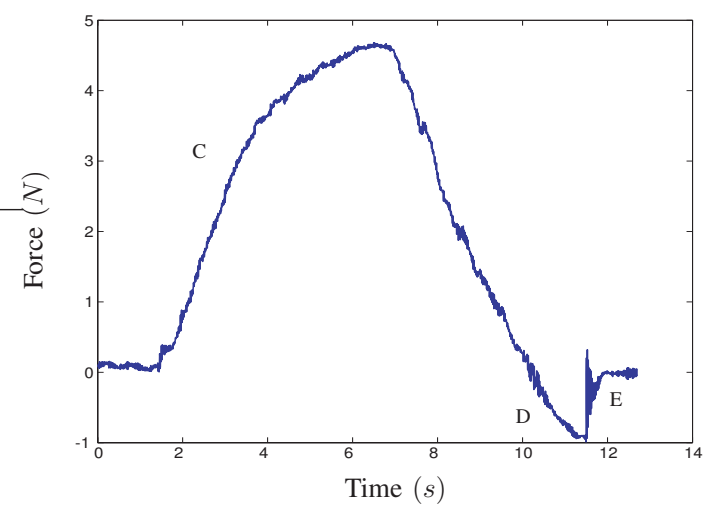

Fig. 9. Approach-retract curve for a cantilever of stiffness $k_{c}=$ $2.4 N . m^{-1}$, using $A_{d}=0.05 \cdot 10^{6}, A_{f}=6 \cdot 10^{6}$. FP control scheme. 
with respect to environment's stiffness, we performed the same experiment using different cantilevers. The results are presented on figure 10 . We required the same velocity-scaling factor for the three probes. The force amplification was chosen so that the user could clearly feel the contact (about $5 N$ are fed back via the haptic device), for a cantilever's deflection of less than $5 \mu \mathrm{m}$. This lead to $A_{f}=200.10^{6}$ for the cantilever of stiffness $k_{c}=0.05 N . m^{-1}$, and $A_{f}=$ $0.2 .10^{6}$ for that of stiffness $k_{c}=48 N . m^{-1}$. Other gains were chosen using the methodology described in section IVB.

Plots on figure 10 show that the operator was able to feel the contact in a stable way for cantilevers of different stiffnesses (from 0.05 up to $48 \mathrm{~N} \cdot \mathrm{m}^{-1}$ ). It should be noted that

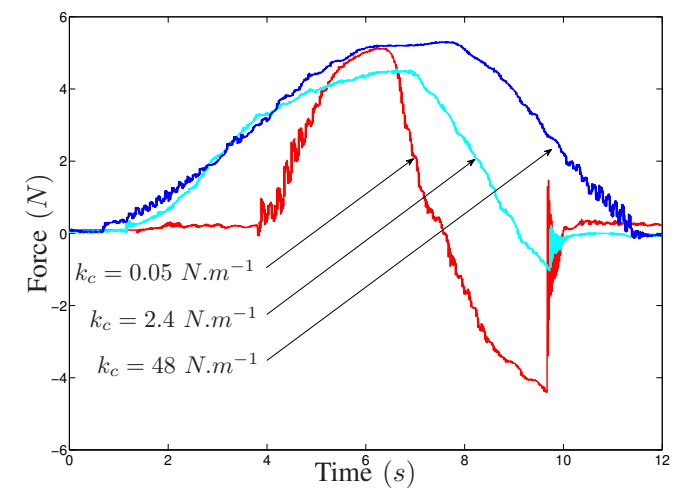

Fig. 10. Approach-retract curve for cantilevers of stiffnesses $k_{c}=$ $\{0.05,2.4,48\} N \cdot m^{-1}\left(K_{f p}=\{100,100,100\} N \cdot m^{-1}, B_{f p}=\right.$ $\left.\{1.2,2.0,1.5\} N . s . m^{-1}, G_{n}=\{2329,47.5,1.98\} m . N^{-1} . s^{-1}\right)$.

these experiments have been performed in a non-controlled environment. Conditions of humidity and temperature may have changed between the experiments. However, the pulloff is indeed greater for cantilevers of low stiffness. The FP control scheme seems adapted to the application described in section II-A, in particular since the system is stable, even when in contact with the substrate. Moreover, the methodology to tune the gains is validated through experiments. Indeed, it allowed us to feel forces of $10 n N$ (pull-off for the cantilever of stiffness $k_{c}=0.05 \mathrm{~N} . \mathrm{m}^{-1}$ ).

However, for the same reasons as in paragraph III-B, the pull-in phenomenon is not visible on the plot.

\section{CONCLUSION}

Two different control schemes designed for nanorobotic applications have been presented in this paper. The Direct Force Feedback scheme suffers from a trade-off between force amplification and workspace limitation. To overcome this problem, the Force-Position control scheme is introduced. Compared to the DFF control scheme, damping is added. It improves stability but degrades transparency. Using the FP control scheme, we were able to feel forces of $10 \mathrm{nN}$. Moreover, stable contact for cantilevers of stiffnesses from $0.05 N . m^{-1}$ to $48 N . m^{-1}$ is demonstrated. Since performances of haptic couplings greatly depend on the tuning of the gains, the choice of these values is a critical issue. Therefore, an original method is applied to tune the gains of these two controllers. Intrinsic limitations of the couplings are exposed theoretically and experimentally.

In future work, we expect to use force feedback to develop original assistance for more complex telemanipulations. Two main tasks need to be performed to reach this goal. The first is to determine how to use the photodiode's outputs to retrieve more information than is presented in this paper (i.e., contact or non-contact). The second is to find new sources of information, such as modifications of objectinduced vibrations of excited cantilevers.

\section{ACKNOWLEDGMENT}

This work was supported by the French National Agency of Research, through the PACMAN project.

\section{REFERENCES}

[1] M. Sitti, "Microscale and nanoscale robotics systems [grand challenges of robotics]," IEEE Robotics and Automation Magazine, vol. 14, no. 1, pp. 53-60, March 2007.

[2] R. Hollis, S. Salcudean, and D. Abraham, "Toward a tele-nanorobotic manipulation system with atomic scale force feedback and motion resolution," in Proceedings of the IEEE International Conference on Micro Electro Mechanical Systems, Feb. 1990, pp. 115-119.

[3] Y. Hatamura and H. Morishita, "Direct coupling system between nanometer world and human world," in Proceedings of the IEEE International Conference on Micro Electro Mechanical Systems, Feb. 1990, pp. 203-208.

[4] A. Ferreira and C. Mavroidis, "Virtual reality and haptics for nanorobotics," IEEE Robotics and Automation Magazine, vol. 13, no. 3, pp. 78-92, Sept. 2006.

[5] M. Sitti and H. Hashimoto, "Teleoperated touch feedback from the surfaces at the nanoscale: modeling and experiments," IEEE/ASME Transactions on Mechatronics, vol. 8, no. 2, pp. 287-298, June 2003.

[6] M. Guthold, M. Falvo, W. Matthews, S. Paulson, S. Washburn, D. Erie, R. Superfine, F. J. Brooks, and R. I. Taylor, "Controlled manipulation of molecular samples with the nanomanipulator," IEEE/ASME Transactions on Mechatronics, vol. 5, no. 2, pp. 189-198, June 2000.

[7] M. Goldfarb, "Dimensional analysis and selective distortion in scaled bilateral telemanipulation," in Proceedings of the IEEE International Conference on Robotics and Automation, May 1998, pp. 1609-1614.

[8] S.-G. Kim and M. Sitti, "Task-based and stable telenanomanipulation in a nanoscale virtual environment," IEEE Transactions on Automation Science and Engineering, vol. 3, no. 3, pp. 240-247, July 2006.

[9] C. D. Onal, C. Pawashe, and M. Sitti, "A scaled bilateral control system for experimental 1-D teleoperated nanomanipulation applications," in Proceedings of the IEEE/RSJ International Conference on Intelligent Robots and Systems, Oct.-Nov. 2007, pp. 483-488.

[10] G. Venture, D. Haliyo, S. Régnier, and A. Micaelli, "Force-feedback micromanipulation with unconditionally stable coupling," in Proceedings of the IEEE/RSJ International Conference on Intelligent Robots and Systems, Aug. 2005, pp. 1923-1928.

[11] H. Xie, J. Vitard, S. Haliyo, S. Régnier, and M. Boukallel, "Calibration of lateral force measurements in atomic force microscopy," Review of Scientific Instrument, vol. 79, p. 033708 (6pp), 2008.

[12] D. Maugis, Contact, adhesion and rupture of elastic solids. Springer, 2000, vol. 130 , ch. 4 , pp. 203-344.

[13] J. J. Gil, A. Avello, A. Rubio, and J. Flórez, "Stability analysis of a 1 DOF haptic interface using the Routh-Hurwitz criterion," IEEE Transactions on Control Systems Technology, vol. 12, no. 4, pp. 583588, July 2004. 